\title{
A note on a problem of Hilliker and Straus
}

\author{
Mirosława Jańczak \\ Faculty of Mathematics and CS \\ Adam Mickiewicz University \\ ul. Umultowska 87, 61-614 Poznań, Poland \\ mjanczak@amu .edu.pl
}

Submitted: May 20, 2006; Accepted: Oct 23, 2007; Published: Oct 30, 2007

Mathematics Subject Classifications: 06124, 06124

\begin{abstract}
For a prime $p$ and a vector $\bar{\alpha}=\left(\alpha_{1}, \ldots, \alpha_{k}\right) \in \mathbb{Z}_{p}^{k}$ let $f(\bar{\alpha}, p)$ be the largest $n$ such that in each set $A \subseteq \mathbb{Z}_{p}$ of $n$ elements one can find $x$ which has a unique representation in the form $x=\alpha_{1} a_{1}+\cdots+\alpha_{k} a_{k}, a_{i} \in A$. Hilliker and Straus [2] bounded $f(\bar{\alpha}, p)$ from below by an expression which contained the $L_{1}$-norm of $\bar{\alpha}$ and asked if there exists a positive constant $c(k)$ so that $f(\bar{\alpha}, p)>c(k) \log p$. In this note we answer their question in the affirmative and show that, for large $k$, one can take $c(k)=O(1 / k \log (2 k))$. We also give a lower bound for the size of a set $A \subseteq \mathbb{Z}_{p}$ such that every element of $A+A$ has at least $K$ representations in the form $a+a^{\prime}, a, a^{\prime} \in A$.
\end{abstract}

\section{Introduction}

Let $f(p)$ denote the largest number $n$ such that in any set $A=\left\{a_{1}, \ldots, a_{n}\right\}$ contained in $\mathbb{Z}_{p}=\mathbb{Z} / p \mathbb{Z}$ at least one difference $a_{i}-a_{j}$ is incongruent to all other differences. Straus [4] estimated $f(p)$ up to a constant factor, showing that

$$
\frac{1}{2} \log _{2}(p-1)+1 \leq f(p)<\frac{(2+o(1))}{\log _{2} 3} \log _{2} p
$$

for all primes $p$. Hilliker and Straus [2] studied the following natural generalization of the problem. For a given vector $\bar{\alpha}=\left(\alpha_{1}, \ldots, \alpha_{k}\right) \in \mathbb{Z}_{p}^{k}$ consider the set of all linear combinations $S=S(\bar{\alpha}, A)=\alpha_{1} A+\alpha_{2} A+\cdots+\alpha_{k} A$. Let $f(\bar{\alpha}, p)$ be the largest $n$ such that for any set $A \subseteq \mathbb{Z}_{p},|A|=n$, one can find at least one element which has the unique representation in $S$. They proved that

$$
f(\bar{\alpha}, p) \geq \frac{\log (p-1)}{\log \left(2\|\bar{\alpha}\|_{1}\right)}+1,
$$


where $\|\bar{\alpha}\|_{1}=\sum_{i=1}^{k}\left|\alpha_{i}\right|$. They ask if the $L_{1}$-norm of a vector $\bar{\alpha}$ can be replaced by a function which depends only on $k$, i.e., if $f(\bar{\alpha}, p)>c(k) \log p$ ?

In the note we settle the above problem in the affirmative (Theorem 1 Corollary 1 below). We also show that our lower bound for $f(\bar{\alpha}, p)$ given in Theorem 1 cannot be much improved (Theorem 2). In section 3 we find a lower bound on $|A \pm A|$ for special sets $A$ such that every element $x \in A+A$ has at least two different representations $a+a^{\prime}$, $a, a^{\prime} \in A$. Finally, we give a lower bound for the size of a set $A \subseteq \mathbb{Z}_{p}$ such that every element $t \in A+A$ has at least $K \geq 2$ representations of the form $t=a+a^{\prime}, a, a^{\prime} \in A$.

Throughout the note $\bar{\alpha}=\left(\alpha_{1}, \alpha_{2}, \ldots, \alpha_{k}\right)$ denotes a vector with nonzero integral components, and $l$ denote the number of different components of $\bar{\alpha}$. By $\log x$ we always mean $\log _{2} x, p$ is a prime, and $A$ is a set of residues modulo $p$. We set $r \cdot T=\{r t: t \in T\}$ but sometimes we shall omit the dot writing for instance $\alpha_{i} A$ instead $\alpha_{i} \cdot A$. By $S=S(\bar{\alpha}, A)$ we mean the set

$$
S=S(\bar{\alpha}, A)=\alpha_{1} A+\alpha_{2} A+\cdots+\alpha_{k} A,
$$

and for a natural $k$ we put

$$
k A=\underbrace{A+A+\cdots+A}_{k} .
$$

For $x \in \mathbb{Z}_{p}$ let $\nu_{\bar{\alpha}}(x)=\nu_{\bar{\alpha}, A}(x)$ be the number of representation of $x$ in $\mathbb{Z}_{p}$ in the form $x=\alpha_{1} a_{1}+\cdots+\alpha_{k} a_{k}$, where $a_{1}, \ldots, a_{k} \in A$. For $t \in \mathbb{R}$ let $\|t\|$ denotes the distance from $t$ to the nearest integer.

Finally, let us mention a simple but important observation that for every $x, d_{1}, d_{2} \in \mathbb{Z}_{p}$, $d_{1} \neq 0$,

$$
\nu_{\bar{\alpha}, A}(x)=\nu_{\bar{\alpha}, d_{1} A+d_{2}}\left(d_{1} x+d_{2} \sum_{i=1}^{k} \alpha_{i}\right) .
$$

\section{A lower bound for $f(\bar{\alpha}, p)$}

First we present a simple argument which shows that in the inequality $f(\bar{\alpha}, p) \geq \frac{\log (p-1)}{\log \left(2\|\bar{\alpha}\|_{1}\right)}+1$, proved by Hilliker and Straus [2], one can replace the factor $\left(\log \left(\|\bar{\alpha}\|_{1}\right)\right)^{-1}$ by a constant depending only on $k$.

Theorem 1. For every $\bar{\alpha}=\left(\alpha_{1}, \alpha_{2}, \ldots, \alpha_{k}\right)$ we have

$$
f(\bar{\alpha}, p) \geq \frac{\log p}{l \log 2 k}
$$

Proof. Let $A=\left\{a_{1}, \ldots, a_{n}\right\}$ be a set such that for every element $x \in S$ we have $\nu_{\bar{\alpha}}(x) \geq 2$ and $|A|=f(\bar{\alpha}, p)+1$. Let $T=\alpha_{1} A \cup \cdots \cup \alpha_{k} A \subseteq \mathbb{Z}_{p}$. Because of (1) we can and shall assume that $a_{1}=0$.

Dirichlet approximation theorem implies that there exists $r, 0<r<p$, such that for every $x \in T$ we have

$$
\left\|\frac{r x}{p}\right\| \leq p^{-\frac{1}{|T|-1}}
$$


Hence, for all $\alpha_{1} a_{1}+\cdots+\alpha_{k} a_{k} \in S$ we have

$$
\left\|\frac{r\left(\alpha_{1} a_{1}+\cdots+\alpha_{k} a_{k}\right)}{p}\right\| \leq\left\|\frac{r \alpha_{1} a_{1}}{p}\right\|+\cdots+\left\|\frac{r \alpha_{k} a_{k}}{p}\right\| \leq k p^{-\frac{1}{|T|-1}} .
$$

We shall show that

$$
p^{-\frac{1}{T \mid-1}} \geq \frac{1}{2 k}
$$

Indeed, suppose that the above inequality does not hold and $p^{-\frac{1}{|T|-1}}<\frac{1}{2 k}$, so that $r \cdot T \subseteq\left(-\frac{p}{2 k}, \frac{p}{2 k}\right)$. Let $x_{i} \in \alpha_{i} r \cdot A(i=1, \ldots, k)$. Observe, that for every $x_{1}+\cdots+x_{k} \in r \cdot S$ we have

$$
\left\|x_{1}+\cdots+x_{k}\right\|<\left\|x_{1}\right\|+\cdots+\left\|x_{k}\right\|<\frac{1}{2} .
$$

Hence, if $m_{i}(i=1, \ldots, k)$ is the largest element in $\alpha_{i} r \cdot A$ considered as a subset of $\left(-\frac{p}{2 k}, \frac{p}{2 k}\right)$, then, clearly, $m_{1}+m_{2}+\cdots+m_{k}$ has exactly one representation in $S$, because the effect modulo is not possible. Therefore

$$
p^{-\frac{1}{|T|-1}} \geq \frac{1}{2 k}
$$

Hence

$$
|T| \geq \frac{\log p}{\log 2 k}+1
$$

and, since the cardinality of $T$ is at most $l(|A|-1)+1$,

$$
f(\bar{\alpha}, p)+1=|A| \geq \frac{\log p}{l \log 2 k}+1
$$

completing the proof of Theorem 1.

Since $l \leq k$ as an immediate consequence of Theorem 1 we get the following result.

Corollary 1. For any $\bar{\alpha}$

$$
f(\bar{\alpha}, p) \geq \frac{\log p}{k \log 2 k}
$$

From Theorem 1 it follows that, in particular, for $\bar{\alpha}^{(k)}=(1,1, \ldots, 1)$ we have

$$
f\left(\bar{\alpha}^{(k)}, p\right) \geq \frac{\log p}{\log 2 k}
$$

Our next result shows that in general this bound cannot be much improved.

Theorem 2. For every $\varepsilon>0, k \geq 2$ and every prime $p>p_{\varepsilon}$ we have

$$
f\left(\bar{\alpha}^{(k)}, p\right)<\left(\frac{2+3 \varepsilon}{\log (2 k-1)}\right) \log p+3 .
$$


Proof. Our construction of a set $A$ is a straightforward generalization of the one presented in [2]. Put

$$
R=\left\{0, \pm 1, \pm 2, \ldots, \pm z_{k}\right\}
$$

where

$$
z_{k}=\left\lceil\frac{k(2 k-1)^{m}-1}{k-1}\right\rceil
$$

and $\frac{2 k}{\varepsilon}<m<\log _{2 k-1}\left(\frac{\varepsilon}{k} \log _{2 k-1} p\right)$. Thus, the set $(k-1) R$ consists of all residues modulo $k(2 k-1)^{m}$. We recursively define a descending sequence $a_{1}, a_{2}, \ldots, a_{l}$ setting

$$
\begin{gathered}
a_{1}=(p-r) / k, p \equiv r \quad \bmod k(2 k-1)^{m}, r \in(k-1) R, \\
a_{i+1}=\left\{\begin{array}{lll}
a_{i} /(2 k-1) & \text { if } a_{i} \equiv 0 \quad \bmod (2 k-1) \\
\left(a_{i}-r_{i}\right) / k & \text { if } a_{i} \neq \equiv 0 \quad \bmod (2 k-1),
\end{array}\right.
\end{gathered}
$$

where $r_{i} \equiv a_{i} \bmod k(2 k-1)^{m}$. The last element $a_{l}$ of this sequence satisfies

$$
a_{l} \geq z_{k}+1, a_{l+1} \in R \text {. }
$$

Define

$$
A=R \cup\left\{ \pm a_{1}, \ldots, \pm a_{l}\right\} .
$$

We need to show that every element $x \in S$ has at least two different representations. It is clear that if $z=a_{1}+\cdots+a_{i}+\cdots+a_{j}+\cdots+a_{k}$ with $a_{i} \neq a_{j}$, then $z=a_{1}+\cdots+a_{j}+$ $\cdots+a_{i}+\cdots+a_{k}$ is another representation of $z$. It remains to show that each element $k a$, where $a \in A$, has at least two representations in $S$. If $a=0$ then it is indeed the case, since

$$
k a=0+\cdots+0=1+(-1)+0+\cdots+0 .
$$

For $0<a<z_{k}$ we have

$$
k a=(a-1)+(a+1)+\underbrace{a+\cdots+a}_{k-2} .
$$

Finally, if $a=z_{k}$, then by (3) and (4)

$$
z_{k}+1 \leq a_{l} \leq(2 k-1) z_{k} .
$$

Hence

$$
(k-1) z_{k}-1 \geq k a-a_{l} \geq-(k-1) z_{k} .
$$

Observe that $k a-a_{l} \in(k-1) R$. So, there exist $b_{1}, \ldots, b_{k-1} \in R$ such that $k a=a_{l}+b_{1}+\cdots+b_{k-1}$.

Now we show that every element $k a_{j}$ has at least two representations in $S$. If $j \geq 2$, then by construction of the sequence we have either $a_{j}=a_{j-1} /(2 k-1)$, or $a_{j}=\left(a_{j-1}-r_{j-1}\right) / k$. If $a_{j}=a_{j-1} /(2 k-1)$, then $(2 k-1) a_{j}=a_{j-1}$ and

$$
k a_{j}=a_{j-1}-(k-1) a_{j}=a_{j-1}+(k-1)\left(-a_{j}\right) .
$$


If $a_{j}=\left(a_{j-1}-r_{j-1}\right) / k$, then

$$
k a_{j}=a_{j-1}+\underbrace{\left(-r_{j-1}\right)}_{\in(k-1) R} .
$$

If $j=1$ then $k a_{1}$ has the following two representations in $S$ :

$$
\begin{gathered}
a_{1}=(p-r) / k, \text { where } r \equiv p\left(\bmod k(2 k-1)^{m}\right), \text { and } r \in(k-1) R, \\
k a_{1}=p-r \equiv-r(\bmod p) .
\end{gathered}
$$

It means that

$$
k a_{1}=\underbrace{a_{1}+\cdots+a_{1}}_{k}=0+\underbrace{(-r)}_{\in(k-1) R} .
$$

Finally, we estimate the cardinality of $A$. Note that

$$
|A|=2 l+2 z_{k}+1=2 l+2\left\lceil\frac{k(2 k-1)^{m}-1}{k-1}\right\rceil+1<2 l+2 \frac{k(2 k-1)^{m}}{k-1}+3 .
$$

Observe that $a_{i+1}<a_{i}$ for all $i$ and $a_{i+1}=a_{i} /(2 k-1)$ for all except at most one out of every $m+1$ consecutive terms $a_{j}, a_{j+1}, \ldots, a_{j+m}$. We have also $a_{j+1} \leq a_{j} / k$ if $a_{j+1}=\left(a_{j}-r_{j}\right) / k$, where $r_{j} \equiv a_{j}\left(\bmod k(2 k-1)^{m}\right), r_{j} \in(k-1) R$. Thus

$$
a_{j+m+1}<k^{-1} a_{j}(2 k-1)^{-m}
$$

and

$$
\frac{k(2 k-1)^{m}}{k-1} \leq a_{l}<p k^{\frac{-l-1}{m+1}}(2 k-1)^{1-\frac{l m}{m+1}}
$$

Hence

$$
l<\frac{1}{m}\left(1-m^{2}+(m+1) \frac{\log p}{\log (2 k-1)}\right)<(1+1 / m) \frac{\log p}{\log (2 k-1)} .
$$

Consequently,

$$
\begin{aligned}
|A| & <2(1+1 / m) \frac{\log p}{\log (2 k-1)}+2 \frac{k(2 k-1)^{m}}{k-1}+3 \\
& <2(1+\varepsilon /(2 k)) \frac{\log p}{\log (2 k-1)}+2 \varepsilon /(k-1) \frac{\log p}{\log (2 k-1)}+3 \\
& =\left(2+\frac{3 k-1}{k(k-1)} \varepsilon\right) \frac{\log p}{\log (2 k-1)}+3 \\
& \leq(2+3 \varepsilon) \frac{\log p}{\log (2 k-1)}+3
\end{aligned}
$$

for $\frac{2 k}{\varepsilon}<m<\log _{2 k-1}\left(\frac{\varepsilon}{k} \log _{2 k-1} p\right)$ and $k \geq 2$.

Next result shows that for each $\alpha$ the order of magnitude of $f(\bar{\alpha}, p)$ is at $\operatorname{most} \log ^{2} p$. This improves the upper bound for $f(\bar{\alpha}, p)$ in [2]. 
Theorem 3. For every $\bar{\alpha}=\left(\alpha_{1}, \ldots, \alpha_{k}\right)$ we have

$$
f(\bar{\alpha}, p) \leq 4 \log ^{2} p
$$

Proof. Observe that if $\bar{\alpha}=\left(1, \alpha_{2}\right)$ and $\bar{\alpha}^{\prime}=\left(1, \alpha_{2}, \alpha_{3}, \ldots, \alpha_{k}\right)$, then $f(\bar{\alpha}, p) \geq f\left(\bar{\alpha}^{\prime}, p\right)$. Let $S$ be a set such that for every element $x \in S+S$ we have $\nu_{(1,1)} \geq 2$ and $|S| \leq 2 \log p$. Let $a_{1}, a_{2} \in A=S+\alpha_{2} S$. Then

$$
\begin{aligned}
a_{1}+\alpha_{2} a_{2} & =\left(s_{1}+\alpha_{2} s_{2}\right)+\alpha_{2}\left(s_{3}+\alpha_{2} s_{4}\right) \\
& =s_{1}+\alpha_{2}\left(s_{2}+s_{3}\right)+\alpha_{2}^{2} s_{4} \\
& =s_{1}+\alpha_{2}\left(s_{2}^{\prime}+s_{3}^{\prime}\right)+\alpha_{2}^{2} s_{4} \\
& =\left(s_{1}+\alpha_{2} s_{2}^{\prime}\right)+\alpha_{2}\left(s_{3}^{\prime}+\alpha_{2} s_{4}\right) \\
& =a_{1}^{\prime}+\alpha_{2} a_{2}^{\prime}
\end{aligned}
$$

for some $a_{1}, a_{2}, a_{1}^{\prime}, a_{2}^{\prime} \in A$ and $s_{1}, s_{2}, s_{3}, s_{4}, s_{2}^{\prime}, s_{3}^{\prime} \in S$. Thus

$$
f(\bar{\alpha}, p) \leq|A| \leq|S|^{2} \leq 4 \log ^{2} p .
$$

\section{The cardinality of sumsets}

In this section we estimate the cardinality of $A-B$, where $A$ is such that every element of $A+A$ has at least two representations, and $B$ is an arbitrary subset of $\mathbb{Z}_{p}$. The main result of this section can be stated as follows.

Theorem 4. If $A \subseteq \mathbb{Z}_{p}$ and for any element $x \in A+A$ we have $\nu_{(1,1)}(x) \geq 2$, then for any $B \subseteq \mathbb{Z}_{p}$

$$
|A-B| \geq|B|\left(\frac{\log p}{\log 12}-|B|\right) .
$$

Proof. Our argument is based on the following result of Ruzsa [3].

Lemma 1. Let $A, B \subseteq G$ be finite sets and $G$ be an abelian group. Then there exists a set $X \subseteq G$ such that $B \subseteq X+A-A$ and $|X| \leq \frac{|B-A|}{|A|}$.

Let $X$ be a set whose existence is guaranteed by Lemma 1, i.e.,

$$
|X| \leq \frac{|A-B|}{|B|} \text { and } A \subseteq X+B-B
$$

By Dirichlet's theorem applied to the set $X \cup B$ there is an integer $0<r<p$ such that for any element $z \in X \cup B$

$$
\left\|\frac{r z}{p}\right\| \leq p^{-\frac{1}{|X|+|B|}}
$$


For every $a \in A$ there exist $b_{1}, b_{2} \in B$ and $x \in X$ such that $a=x+b_{1}-b_{2}$. Hence

$$
\left\|\frac{r a}{p}\right\| \leq\left\|\frac{r x}{p}\right\|+\left\|\frac{r b_{1}}{p}\right\|+\left\|\frac{r b_{2}}{p}\right\| \leq 3 p^{-\frac{1}{|X|+|B|}} .
$$

Moreover, arguing as in the proof of Theorem 1 (cf. (2)), we get

$$
3 p^{-\frac{1}{|X|+|B|}} \geq \frac{1}{4}
$$

Thus

$$
|X| \geq \frac{\log p}{\log 12}-|B|
$$

and, from (5),

$$
|A-B| \geq|B||X| \geq|B|\left(\frac{\log p}{\log 12}-|B|\right) .
$$

Corollary 2. If $A \subseteq \mathbb{Z}_{p}$ and for any element $x \in A+A$ we have $\nu_{(1,1)}(x) \geq 2$, then

$$
|A \pm A| \geq\left\lfloor\frac{\log p}{2 \log 12}\right\rfloor^{2}
$$

Proof. Pick any set $B \subseteq \pm A$ with $|B|=\left\lfloor\frac{\log p}{2 \log 12}\right\rfloor$ and apply Theorem 4 for the sets $A$ and $B$.

Let $f_{K}(p)$ be the largest $n$ such that for any set $A \subseteq \mathbb{Z}_{p}$ with at most $f_{K}(p)$ elements there exists at least one element in $A+A$ with less then $K$ representations. As a corollary from Theorem 4 we obtain the following lower bound for $f_{K}(p)$.

Corollary 3. For every $K \geq 2$ we have

$$
f_{K}(p) \geq \sqrt{K}\left\lfloor\frac{\log p}{2 \log 12}\right\rfloor-1 .
$$

Proof. Let us assume that $A \subseteq \mathbb{Z}_{p}$, for each element $x \in A+A$ we have $\nu_{(1,1)}(x) \geq K \geq 2$, and $|A|=f_{K}(p)+1$. By Corollary 2 we get

$$
|A+A|>\left\lfloor\frac{\log p}{2 \log 12}\right\rfloor^{2} .
$$

Since

$$
K|A+A| \leq \sum_{t \in A+A} \nu_{(1,1)}(t)=|A|^{2}
$$

it follows that

$$
\frac{|A|^{2}}{K} \geq|A+A|
$$


From (6) and (7), we get

$$
f_{K}(p)+1=|A| \geq \sqrt{K}\left\lfloor\frac{\log p}{2 \log 12}\right\rfloor,
$$

and so

$$
f_{K}(p) \geq \sqrt{K}\left\lfloor\frac{\log p}{2 \log 12}\right\rfloor-1 .
$$

\section{References}

[1] J. Browkin, B. Diviš, A. Schinzel, Addition of sequences in general fields, Monatshefte für Mathematik 82 (1976), 261-268.

[2] D. L. Hilliker, E. G. Straus, Uniqueness of linear combinations ( $\bmod p)$, Journal of Number Theory 24 (1986), 1-6.

[3] I. Z. RuZsA, An analog of Frieman's theorem in groups, Asterisque 258 (1999), $323-326$.

[4] E. G. Straus, Differences of residues $(\bmod p)$, Journal of Number Theory 8 (1976), $40-42$. 